\title{
Cordycepin Increases Nonrapid Eye Movement Sleep via Adenosine Receptors in Rats
}

\author{
Zhenzhen Hu, ${ }^{1,2}$ Chung-Il Lee, ${ }^{1}$ Vikash Kumar Shah, ${ }^{1}$ Eun-Hye Oh, ${ }^{1}$ Jin-Yi Han, ${ }^{3}$ \\ Jae-Ryong Bae, ${ }^{4}$ Kinam Lee, ${ }^{4}$ Myong-Soo Chong, ${ }^{4}$ Jin Tae Hong, ${ }^{1}$ and Ki-Wan $\mathrm{Oh}^{1}$ \\ ${ }^{1}$ College of Pharmacy, Chungbuk National University, Cheongju 361-763, Republic of Korea \\ ${ }^{2}$ Department of Pathophysiology, College of Medicine, Nanchang University, Nanchang, Jiangxi 33006, China \\ ${ }^{3}$ Research Institute of Veterinary Medicine, Chungbuk National University, Cheongju 361-763, Republic of Korea \\ ${ }^{4}$ College of Oriental Medicine, Wonkwang University, Iksan 570-749, Republic of Korea \\ Correspondence should be addressed to Ki-Wan Oh; kiwan@chungbuk.ac.kr
}

Received 6 December 2012; Revised 22 March 2013; Accepted 29 March 2013

Academic Editor: Shao Li

Copyright (C) 2013 Zhenzhen $\mathrm{Hu}$ et al. This is an open access article distributed under the Creative Commons Attribution License, which permits unrestricted use, distribution, and reproduction in any medium, provided the original work is properly cited.

Cordycepin ( $3^{\prime}$-deoxyadenosine $)$ is a naturally occurring adenosine analogue and one of the bioactive constituents isolated from Cordyceps militaris/Cordyceps sinensis, species of the fungal genus Cordyceps. It has traditionally been a prized Chinese folk medicine for the human well-being. Because of similarity of chemical structure of adenosine, cordycepin has been focused on the diverse effects of the central nervous systems (CNSs), like sleep regulation. Therefore, this study was undertaken to know whether cordycepin increases the natural sleep in rats, and its effect is mediated by adenosine receptors (ARs). Sleep was recorded using electroencephalogram (EEG) for 4 hours after oral administration of cordycepin in rats. Sleep architecture and EEG power spectra were analyzed. Cordycepin reduced sleep-wake cycles and increased nonrapid eye movement (NREM) sleep. Interestingly, cordycepin increased $\theta$ (theta) waves power density during NREM sleep. In addition, the protein levels of $A R$ subtypes $\left(A_{1}, A_{2 A}\right.$, and $A_{2 B}$ ) were increased after the administration of cordycepin, especially in the rat hypothalamus which plays an important role in sleep regulation. Therefore, we suggest that cordycepin increases theta waves power density during NREM sleep via nonspecific $\mathrm{AR}$ in rats. In addition, this experiment can provide basic evidence that cordycepin may be helpful for sleep-disturbed subjects.

\section{Introduction}

Purines are ubiquitous molecules with important roles in the regulation of metabolic networks and signal transduction events. In the central nervous systems (CNSs), adenosine and ATP modulate the sleep-wake cycles, acting as ligands of specific transmembrane receptors and as allosteric effectors of key intracellular enzymes for brain energy expenditure [1]. Adenosine levels are influenced by neuronal activity. Adenosine is a secondary by-product of the breakdown of ATP and cAMP. When ATP is coreleased with neurotransmitters, ectonucleotidases in the extracellular space can rapidly dephosphorylate ATP, ADP, and AMP into adenosine. ATP release from astrocytes also contributes to extracellular levels of adenosine that have a powerful modulatory effect on synaptic transmission [2]. The role of this astrocyte-derived adenosine in sleep-waking homeostasis was recently investigated. Further research showed that systemic administration of adenosine, its analogs, or inhibitors of its metabolism increase nonrapid eye movement (NREM) sleep in rodents, especially [3].

The local administration of adenosine and/or adenosine receptors (ARs) agonists into the medial preoptic area of hypothalamus, magnocellular cholinergic basal forebrain, brainstem cholinergic areas, the laterodorsal and pedunculopontine tegmental nuclei (LDT/PPT), and pontine reticular formation leads to sleep or reduction of wakefulness [4-6]. In the basal forebrain, both cholinergic and noncholinergic neuronal activities are associated with promoting wakefulness [7]. The somnogenic effects of adenosine may be due to the inhibition of neuronal activity in both cholinergic and noncholinergic neurons of the basal forebrain. In addition, the 
modulatory effects of sleep deprivation on the $A_{1} R$ mRNA in the cholinergic basal forebrain suggest the significance of an adrenergic pathway in the long-term effects of sleep deprivation on the quality of ensuring sleep [8]. Cholinergic neurons of LDT and PPT also comprise the cholinergic "arousal system." During the transition from waking to sleep, the firing rate of LDT/PPT neurons markedly is decreased, reducing the cholinergic tone of their target sites and thus facilitating the transition to sleep [9]. In particular, the ventrolateral preoptic area of the hypothalamus contains a population of sleep-active neurons and is hypothesized to be an important part of the somnogenic process [10].

The four adenosine forms make up the family of Gprotein-coupled AR: $A_{1}, A_{2 A}, A_{2 B}$, and $A_{3}$ [11]. The difference between the four ARs is found in their affinity for adenosine, in the type of $G$ proteins that they associate with, and the signaling pathways that are activated in the target cells. For sleep-waking homeostasis, $\mathrm{A}_{1} \mathrm{R}$ and $\mathrm{A}_{2 \mathrm{~A}} \mathrm{R}$ have received the most attention due to their expression pattern in the nervous system, the availability of selective agonists, and antagonists and selective molecular lesions of genes encoding the receptor subtypes. $\mathrm{A}_{1}$ and $\mathrm{A}_{3}$ Rs have high and low affinity for adenosine [12]. Possible changes in adenosine functioning due to the aging process have been observed in animal models, and abnormalities in the adenosine system could also explain primary insomnia or the reduced amount of delta waves sleep and increased sensitivity to caffeine in some subjects with sleep deficits. Caffeine is a methylated derivate of xanthine with profound effects on the onset and quality of sleep episodes [13]. This purine acts principally as an antagonist of the $\mathrm{A}_{2 \mathrm{~A}} \mathrm{R}$. Adenosine and ATP in the nervous systems are the bridge between metabolic activity, recovery function, and purinergic transmission that underlies the daily wake-sleep cycle in mammals. Modulators of purine actions have the potential to alleviate insomnia and other sleep disorders based on their physiopathological role during the sleep process [14].

Cordycepin $\left(3^{\prime}\right.$-deoxyadenosine, Figure 1$)$ is a naturally occurring adenosine analogue and one of the bioactive constituents of Cordyceps sinensis/militaris $[15,16]$. C. sinensis/militaris has been used for hundreds of years as a traditional medicine in treating disorders of the lung and kidney through mechanisms of immunomodulation [17]. Because of similarity of chemical structure of adenosine, cordycepin has been interested in the diverse effects of CNS, like sleep regulation. It also has traditionally been focused on the treatment of insomnia. In particular, cordycepin is known to be a bioactive constituent to regulate homeostatic function [18]. Recently, some herbs have been the charming medicines for considerable sufferers with sleep disabilities or insomnia [19]. Most remedies of sleep aid originated from herbs have been targeted on $\mathrm{GABA}_{\mathrm{A}}$ systems. However, recent morphological and functional studies have identified AR in the ventrolateral preoptic areas of the hypothalamus that plays an important role in sleep regulation [20]. Therefore, this study was designed to know whether cordycepin increases sleep via $A R$. This experiment also can provide basic evidence that cordycepin may be helpful for the treatment of insomnia.

\section{Materials and Methods}

2.1. Experimental Animals. Male Wistar rats (Samtako, Osan, Korea) weighing 250-300 g were used for sleep recording and western blot. Each rat was housed in each acrylic cage $(45 \times 60 \times 25 \mathrm{~cm})$ with water and food available ad libitum under an artificial $12 \mathrm{~h}$ light-dark cycle (lights on at 7:00 am) and at constant temperature $\left(22 \pm 2^{\circ} \mathrm{C}\right)$. To ensure adaptation to the new environment, mice and rats were kept in the departmental holding room for 1 week before testing. This study was performed in accordance with the Chungbuk National University Laboratory Animal Research Center guidelines for the care and use of laboratory animals.

2.2. Experimental Procedure. After 7 days postsurgical recovery, cordycepin ( 2 and $4 \mathrm{mg} / \mathrm{kg}$ in distilled saline) was administered to animals for 5 days once per day. On the 5 th day, $1 \mathrm{~h}$ after final cordycepin treatment, animals were caged in recording system, and spontaneous sleeping was recorded.

2.3. Brain Surgery and EEG Recording. Each rat was implanted with a transmitter (Data Sciences International, TAllCTAF40, MN, USA) for recording EEG and activity via telemetry. The body of the transmitter was implanted subcutaneously off the midline and posterior to the scapula and was attached to the skin with 3 sutures for stabilization. Leads from the transmitter led subcutaneously to the skull, and the bare ends were placed in contact with the dura through holes made in the skull (A: 2.0 (Bregma), L: 1.5; P: 7.0 (Bregma), $L: 1.5$ contralateral) [21]. The electrodes were anchored to the skull with screws and dental cement. All surgical procedures were performed stereotaxically under aseptic conditions. Surgical anesthesia was achieved with pentobarbital $(50 \mathrm{mg} / \mathrm{kg}$, i.p), and all efforts were made to minimize the suffering of the animals. Telemetric recording of cortical EEG and activity was conducted using procedures similar to previous reports. For the EEG signal, the gain of transmitters was set at $-0.5 /+0.5$ volts per/units $\times 2$, and the raw signals generated from the transmitter were in the range of $0.5-20.0 \mathrm{~Hz}$. The signals were processed by a Data Sciences International analog converter and routed to an $\mathrm{AD}$ converter (Eagle PC30, USA) housed in a PC computer. The AD converter digitized the EEG and activity signals at $128 \mathrm{~Hz}$. The digitized data were transferred to the computer and displayed graphically. An online fast Fourier transformation (FFT) was performed on the EEG data at $10 \mathrm{sec}$ intervals during data acquisition (1024 samples) after a Hanning window treatment. The FFT analysis generated power density values from 0.0 to $20.0 \mathrm{~Hz}$ at a resolution of $0.5 \mathrm{~Hz}$. The FFT data were further averaged in the range of 0 to $20 \mathrm{~Hz}$ for every $10 \mathrm{sec}$. The sleep data and FFT results were saved to the hard disk every $10 \mathrm{sec}$ for additional offline analysis. Movement of the animal in relation to the telemetry receiver generated transistor-transistor logic (TTL) pulses that were collected and counted as a measure of activity. Cordycepin was administered 1 hour before the EEG recording. Recording began at 9:00 am for $4 \mathrm{~h}$. Seven or eight rats were used in each group. 
<smiles>Nc1ncnc2c1ncn2[C@@H]1O[C@H](CO)C[C@H]1O</smiles>

Cordycepin<smiles>Nc1ncnc2c1ncn2[C@@H]1O[C@H](CO)[C@H](O)[C@H]1O</smiles>

Adenosine<smiles>Cn1c(=O)c2c(ncn2C)n(C)c1=O</smiles>

Caffeine

FIGURE 1: Chemical structures of cordycepin, adenosine, and caffeine.

2.4. Analysis of Sleep Architecture. The amounts of time in wakefulness, NREM, and REM sleep were determined from the digitized data at $10 \mathrm{sec}$ intervals using sleep analysis software, SleepSign 2.1 (KISSEI Comtec Co. Ltd., Matsumoto, Japan). Briefly, the software discriminates wakefulness as high-frequency, low-amplitude EEG. NREM was scored based on the presence of spindles interspersed with slow waves in the EEG. EEG power during REM is significantly reduced in lower frequency $\delta$-wave $(0.75-4.0 \mathrm{~Hz})$ and increased in the range of $\theta$-wave activity $(5.0-9.0 \mathrm{~Hz}$, peak at $7.5 \mathrm{~Hz}$ ). The time spent (min) in NREM, REM, total sleep time (NREM + REM), and numbers of sleep-wake cycle were processed to obtain $4 \mathrm{~h}$ period totals for each rat. We further calculated the time of each recording spent in the sleep-wake state (wake, NREM, and REM). The absolute EEG power during wakefulness, NREM, and REM were calculated in $0.5 \mathrm{~Hz}$ bins from 0.5 to $20 \mathrm{~Hz}$ for the entire $4 \mathrm{~h}$ reading of each recording process [22]. Data from seven or eight rats in each group were analyzed.

2.5. Membrane Protein Preparation. After deep anesthesia (induced by diethyl ether), animals were decapitated, and the brain was quickly removed and chilled in ice cold saline. Coronal sections were made using a Rodent Brain Matrix (ASI Instruments). This was immediately followed by a $1,200 \mu \mathrm{m}$ section containing the medial basal hypothalamus, and samples were immediately frozen on dry ice and stored at $-80^{\circ} \mathrm{C}$ [23]. Frozen tissue samples were homogenized in PRO-PREPTM protein extraction solution (iNTRON Biotechnology, Inc.). The homogenate was centrifuged at $15,000 \mathrm{~g}$ at $4^{\circ} \mathrm{C}$ for $20 \mathrm{~min}$, and the supernatant was recovered. A portion of the supernatant was collected to determine the protein concentration and for western blot analysis. The concentration of total protein was determined by a modified Lowry method using bovine serum albumin as a standard. The samples were stored at $-20^{\circ} \mathrm{C}$.

2.6. Western Blot. The total proteins of hypothalamus were loaded in each lane, and sodium dodecyl sulphate polyacrylamide gel electrophoresis (SDS/PAGE) was performed using $12 \%$ polyacrylamide gels. Proteins were transferred to PVDF membranes (Amersham Hybond-P, GE Healthcare) using a semidry transfer system. Receptors were detected with the following primary antibodies: rabbit antiadenosine receptor $\mathrm{A}_{1}$ (diluted 1:1000 in PBS containing 0.5\% Tween
20, Abcam), mouse antiadenosine receptor $\mathrm{A}_{2 \mathrm{~A}}$ (diluted $1: 1000$ in PBS containing $0.5 \%$ Tween 20), and goat antiadenosine receptor $\mathrm{A}_{2 \mathrm{~B}}$ (diluted 1:1000 in PBS containing $0.5 \%$ Tween 20 ). The following horseradish peroxidase secondary antibodies were used: goat antrabbit IgG (diluted $1: 5000)$, rabbit antimouse IgG, and antigoat IgG (1:5000). After stripping, membranes were developed with rabbit anti-GAPDH (1:1000; Santa Cruz Biotechnology Inc, USA) followed by goat antirabbit IgG to confirm equal protein loading. Immunoreactive bands were developed with a BM Chemiluminescence Detection Kit (Roche Diagnostics). Quantitative analysis of detected bands was performed with densitometric scanning, and all values were normalized using GAPDH as a standard [24].

2.7. Statistical Analysis. All data were analyzed using SPSS 17.0 software (SPSS). Significant differences after one-way ANOVAs were measured by post hoc Holm-Sidak test. $P<$ 0.05 was considered to be significant. The values are expressed as mean \pm SEM. All statistical analyses were conducted using SigmaStat software.

\section{Results}

3.1. Effects of Cordycepin on the Number of Sleep-Wake Cycles. Cordycepin $(2 \mathrm{mg} / \mathrm{kg}, P<0.05)$ and $(4 \mathrm{mg} / \mathrm{kg}, P<$ 0.01 ) significantly reduced the number of sleep-wake cycles, respectively, compared with that of the control (Figure 2).

3.2. Effects of Cordycepin on Sleep Architecture. Cordycepin ( 2 and $4 \mathrm{mg} / \mathrm{kg})$ significantly increased NREM $(P<0.005$ and $P<0.05)$ and decreased REM sleep $(P<0.01$ and $P<$ $0.01)$. However, both wakefulness and total sleep time were not changed significantly, compared with that of the control (Figure 3).

3.3. Effects of Cordycepin on EEG Power Density during Total Sleep Time. No significant changes in delta wave, theta wave, and alpha waves power density during total sleep time were observed; cordycepin- (2 and $4 \mathrm{mg} / \mathrm{kg}$ ) treated groups, compared with that of the control (Figure 4).

3.4. Effects of Cordycepin on EEG Power Density during NREM Sleep. Interestingly, cordycepin decreased delta waves 


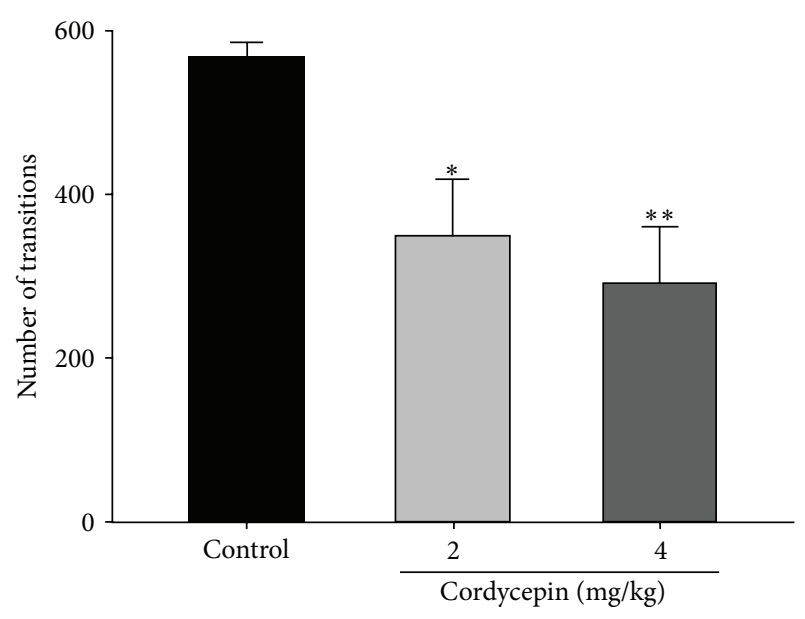

FIGURE 2: Effects of cordycepin (2 and $4 \mathrm{mg} / \mathrm{kg}$ ) on sleep-wake cycles. Values are expressed as the mean \pm SEM. ${ }^{*} P<0.05$ and ${ }^{* *} P<0.01$, compared with that of the control. Five to six animals were used in each group.

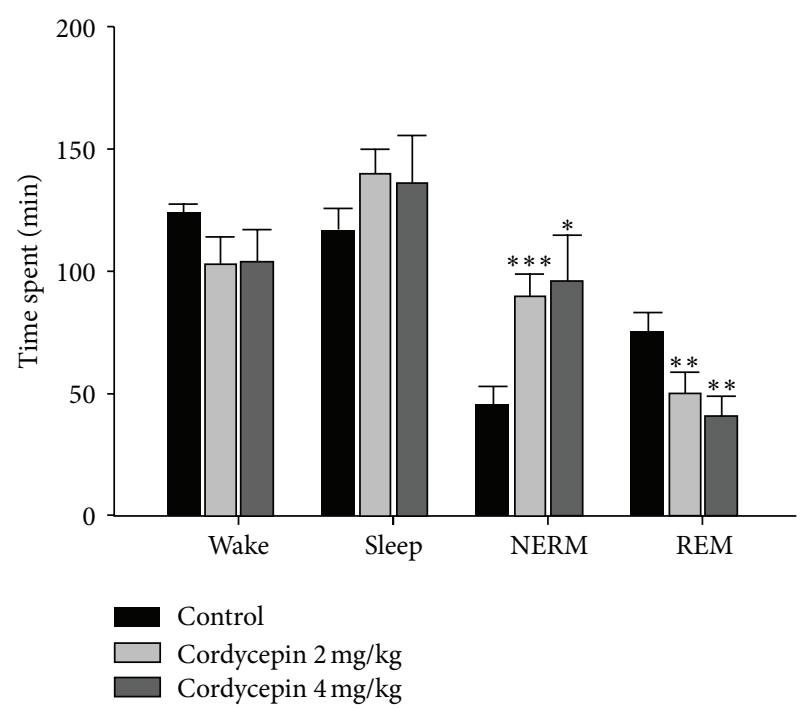

FIGURE 3: Effects of cordycepin ( 2 and $4 \mathrm{mg} / \mathrm{kg}$ ) on rat sleep architecture. Values are expressed as the mean \pm SEM. NREM, nonrapid eye movement; REM, rapid eye movement. ${ }^{*} P<0.05$, ${ }^{* *} P<0.01$, and ${ }^{* * *} P<0.005$, compared with that of the control. For more details, refer Figure 1.

$(P<0.005)$ during NREM sleep. However, theta waves $(P<$ $0.005)$ during NREM sleep significantly were increased by cordycepin $(4 \mathrm{mg} / \mathrm{kg})$. No changes in alpha waves power density during NREM sleep were observed, compared with that of control (Figure 5).

3.5. Effects of Cordycepin on EEG Power Density during REM Sleep. No significant changes were observed in delta wave, theta wave, or alpha wave power density during REM sleep in cordycepin- (2 and $4 \mathrm{mg} / \mathrm{kg}$ ) treated groups, compared with that of the control (Figure 6).

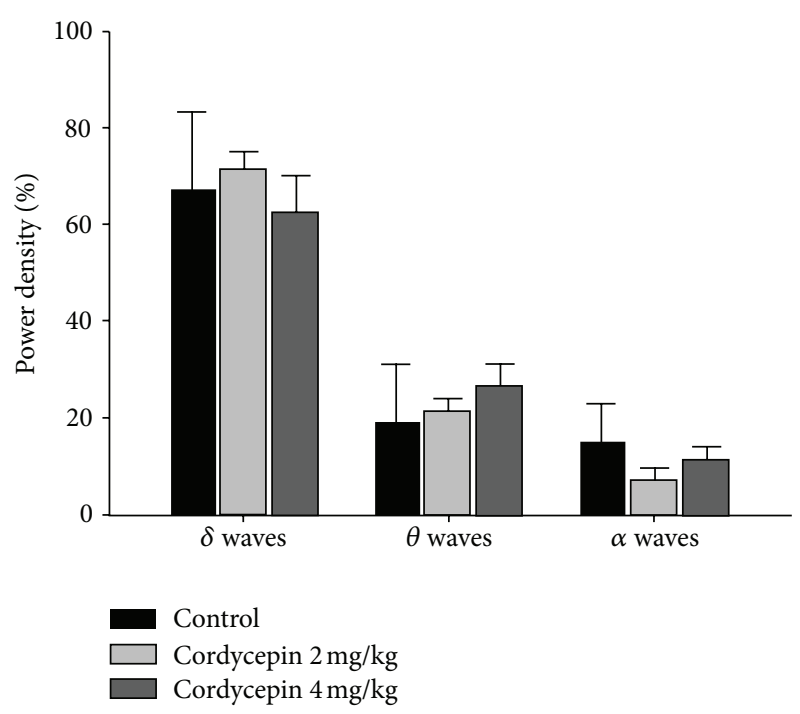

FIGURE 4: Effects of cordycepin (2 and $4 \mathrm{mg} / \mathrm{kg}$ ) treatment on EEG power density during total sleep time. EEG power densities in delta wave, theta wave and alpha wave, spectral bandwidths were evaluated during total sleep time. No significant differences, compared with that of the control. For more details, refer Figure 1.

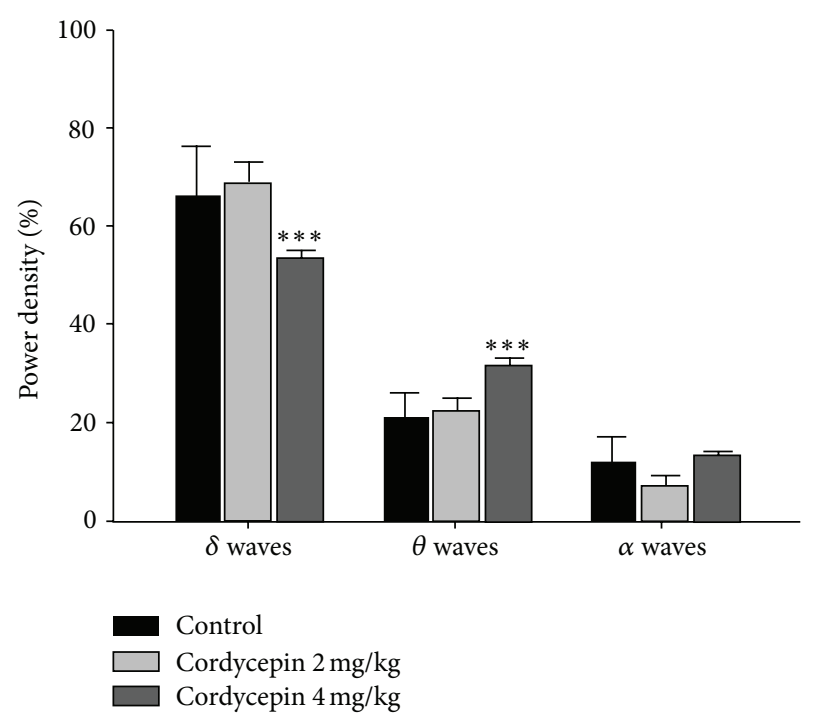

FIgURE 5: Effects of cordycepin (2 and $4 \mathrm{mg} / \mathrm{kg}$ ) on EEG power density during NREM sleep. EEG power densities in delta wave, theta wave, and alpha wave spectral bandwidths were evaluated. Values are expressed as the mean \pm SEM of EEG power densities in three selected frequency bands during NREM sleep. ${ }^{* * *} P<0.005$, compared with that of the control. For more details, refer Figure 1.

3.6. Effects of Cordycepin on the Protein Levels of the $A_{1} R$, $A_{2 A} R$, and $A_{2 B} R$ Subtypes in the Rat Hypothalamus. The protein levels of the $A_{1} R, A_{2 A} R$, and $A_{2 B} R$ subtypes in the hypothalamus were measured by western blot after codycepin administration. Cordyceps $(4 \mathrm{mg} / \mathrm{kg})$ increased the protein levels of $\mathrm{A}_{1} \mathrm{R}, \mathrm{A}_{2 \mathrm{~A}} \mathrm{R}$, and $\mathrm{A}_{2 \mathrm{~B}} \mathrm{R}$ subtypes $\left({ }^{* * *} P<0.005\right.$, ${ }^{* *} P<0.01$, and $\left.{ }^{*} P<0.05\right)$, compared with that of the control (Figure 7). 




FIGURE 6: Effects of cordycepin (2 and $4 \mathrm{mg} / \mathrm{kg}$ ) on EEG power density during REM sleep. EEG power densities in delta wave, theta wave, and alpha wave spectral bandwidths were evaluated. Values are expressed as the mean \pm SEM of EEG power densities in three selected frequency bands for the REM sleep. No significant differences, compared with that of the control. Five to six animals were used in each group. For more details, refer Figure 1.

\section{Discussion}

C. sinensis/militaris which contain cordycepin ( $3^{\prime}$-deoxyadenosine), a naturally occurring adenosine analogue and one of the bioactive constituents, have been used for hundreds of years as traditional medicines in treating insomnia [15]. In this study, cordycepin reduced sleep-wake cycles, increased NREM sleep, and decreased REM sleep. It also increased total sleep time in rodents and decreased wakefulness. Interestingly, power spectral analysis showed that cordycepin significantly increased theta waves power density during NREM sleep. Therefore, it will be suggested that cordycepin, an adenosine analogue, plays a role in the modulation of theta oscillations in NREM sleep. Sleep in most mammals is divided into two major types of sleep, REM sleep and NREM sleep. REM sleep is characterized by fast waves sleep with muscle atonia, activation of brain, and eye movement. NREM sleep characterized by slow waves sleep is emerged in delta waves [25]. During NREM sleep, neuronal activity, metabolic rate, and brain temperature are low. From the EEG experiment, cordycepin increased NREM sleep and reduced REM sleep. However, it tended to increase slow waves in total sleep although they were not significantly increased. On the contrary, theta waves in NREM sleep were significantly increased, delta waves in NREM sleep slightly decreased, and no change in alpha waves was observed. Theta waves between alpha and delta waves in wavelength specially were increased in NREM sleep [26].

Multiple interacting neurotransmitter systems in the brain stem, hypothalamus, and basal forebrain converge onto common effector systems in the thalamus and cortex. Sleep results from the inhibition of wake-promoting systems by

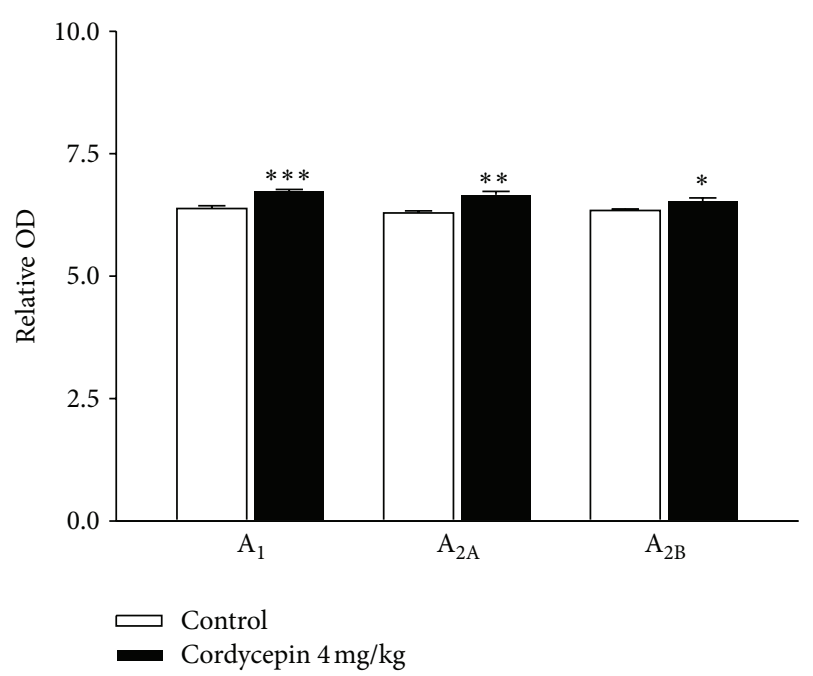

FIgURE 7: Expression of AR: $A_{1}, A_{2 A}$, and $A_{2 B}$ in the rat hypothalamus after cordycepin treatment. AR subtypes in the rat hypothalamus after cordycepin treatment were analyzed by western blotting. The intensity of the immunoreactive bands of 3 to 4 independent experiments was measured by densitometry scanning and normalized using glyceraldehyde 3-phosphate dehydrogenase (GAPDH) as a standard (bar graph). Results are presented as the percentage immunoreactivity detected in the hypothalamus with respect to the GAPDH protein loading control. Values are expressed as the mean \pm SEM. ${ }^{*} P<0.05,{ }^{* *} P<0.01$, and ${ }^{* * *} P<0.005$, compared with that of the control.

homeostatic sleep factors such as adenosine, nitric oxide, and GABAergic neurons in the preoptic area of the hypothalamus, resulting in large-amplitude, slow EEG oscillations [27]. One ubiquitous neuromodulatory system in the CNS is operated by adenosine. Adenosine is an important homeostatic sleep factor acting in basal forebrain and preoptic areas through $A_{1} R$ and $A_{2 A} R$. Recent morphological and functional studies have identified $A R$ in the ventrolateral preoptic areas of the hypothalamus that plays an important role in sleep regulation [28]. Adenosine neuromodulation is mostly conceived as an inhibitory system that restrains excitatory transmission through activation of inhibitory $\mathrm{A}_{1} \mathrm{R}$. Furthermore, adenosine modulates the neuronal activity of basal forebrain and preoptic/anterior hypothalamic in the control of behavioral state [7]. The sleep-inducing effect of adenosine is most efficient when adenosine or its agonists are applied locally into the basal forebrain and preoptic/anterior hypothalamus [29, 30]. The compensatory sleep response to sleep deprivation is significantly reduced when the increase in adenosine or $\mathrm{A}_{2} \mathrm{R}$ activation is blocked [31]. $\mathrm{A}_{2 \mathrm{~A}} \mathrm{R}$ is expressed in the brain areas such as striatum, nucleus accumbens, and olfactory tubercle [32]. Selective $A_{2 A} R$ agonist administration into the subarachnoid space adjacent to the basal forebrain and the lateral preoptic area indduces NREM sleep [33]. In contrast to other brain areas, increases in extracellular adenosine levels in the basal forebrain are very sensitive to even short periods of prolonged waking; adenosine levels continue to rise throughout the waking period and do not decline until sleep is initiated. As the basal forebrain is essential for 
wake promotion, the sleep-inducing effect is thought to be mediated via inhibition of the wake-active basal forebrain cells $[34,35]$. Prolonged waking activates inducible nitric oxide synthase in the basal forebrain, which causes adenosine release and recovery sleep through energy depletion. There are numerous reports indicating that endogenous adenosine is a candidate for the homeostatic sleep factor theory inducting sleep after prolonged wakefulness [35].

Adenosine's pivotal role in sleep modulation is strongly supported by the subjective and EEG-defined arousal produced by its antagonists, caffeine, and theophylline, as well as by the fact that extracellular adenosine concentration is linked to neuronal metabolic activity $[1,36]$. Researches showed that systemic or local administration of adenosine, its analogs, or inhibitors of its metabolism increase especially NREM sleep in rodents [37]. The mechanism by which cordycepin increased NREM sleep and decreased wakefulness was probably achieved through activation of adenosine receptors. Thereby, we suggest that cordycepin mediated effects on sleep-wake states are site and receptor dependent. Furthermore, cordycepin may pharmacologically act in the same manner as that of adenosine as cordycepin has affinity at the $A_{1}, A_{2}$, and $A_{3}$ receptors [38]. For sleep-waking homeostasis, $A_{1} R$ and $A_{2 A} R$ have received the most attention due to their expression pattern in the nervous system. $A_{1} R$ is widely distributed in CNS and inhibits especially cholinergic neurons from forebrain and LDT/PPT [39]. Stimulation of $A_{1} R$ results in adenylate cyclase inhibition and phospholipase $\mathrm{C}$ activation. Higher level of $\mathrm{A}_{1} \mathrm{R}$ agonist or higher expression of $A_{1} R$ potentiates the phospholipase $C$ pathway [40]. The activation of presynaptic $A_{1} R$ inhibits neurotransmitter release, mostly of excitatory neurotransmitter such as glutamate and acetylcholine. From this study, cordycepin activated AR subtypes. So the present report strongly suggests that cordycepin enhances NREM sleep in activating upon AR.

Therefore, administration of cordycepin, an adenosine analogue, widely modulated the power spectral densities of EEG architecture. Increase of total sleep and NREM sleep by cordycepin might be related to the action upon AR. Moreover, the mechanisms which cordycepin modulates EEG architecture and sleep behaviors may be attributed to the similar chemical structure of adenosine. The use of several herbs in the treatment of sleep disorder, such as insomnia, is regarded as a developing market for agents acting on GABA receptors. St. John's Wort, valerian extracts, and ginseng species are the best examples of this issue [41-43]. In addition, ginseng extract enhanced the total sleep and NREM sleep, and reduced the number of sleep-wake cycles [44, 45]. Nevertheless, this is the first experiment that cordycepin, a natural product for the treatment of sleep disorders, such as insomnia, acts on AR.

Cordycepin is quickly deaminated by adenosine deaminase and rapidly metabolized to an inactive metabolite, $3^{\prime}$ deoxyhypoxanthinosine, in vivo [46]. Adenosine is otherwise transported into cells by several nucleoside permeases, and then the adenosine will be phosphorylated to form ATP by adenosine kinase. If the cell does not utilize the adenosine, it will be deaminated to hypoxanthinosine by adenosine deaminase inside the cell [47]. So adenosine and cordycepin had short elimination half-lives and high rates of clearance [18]. We also presume that cordycepin has short-acting compound in the regulation of sleep. Therefore, EEG recording was performed for 4 hours.

\section{Conclusions}

Cordycepin, targeting on AR, can be helpful for sleep disturbance subjects. Thus, the results also indicated a role for cordycepin, an adenosine analogue, in the regulation of sleep and held promise for a new class of compounds as potential agents in the treatment of sleep disorders, such as insomnia. Cordycepin as an adenosine analogue increased NREM sleep and decreased REM sleep, suppressing waking. Cordycepin also increases theta waves power density during NREM sleep via nonspecific $A R$ in the ventrolateral preoptic area of the hypothalamus of the rats. Further research would be necessary to understand the cellular and molecular mechanisms of cordycepin-induced sleep modulation in the brain.

\section{Conflict of Interest}

The authors declare that they have no conflict of interests.

\section{Acknowledgments}

This work was supported by the research grant of Chungbuk National University in 2011 and was kindly gifted from SiKwan Kim, a Professor of Konkuk University.

\section{References}

[1] M. Diaz-Munoz and R. Salin-Pascual, "Purine molecules as hypnogenic factors role of adenosine, ATP, and caffeine," Central Nervous System Agents in Medicinal Chemistry, vol. 10, no. 4, pp. 259-268.

[2] O. Pascual, K. B. Casper, C. Kubera et al., "Neurobiology: astrocytic purinergic signaling coordinates synaptic networks," Science, vol. 310, no. 5745, pp. 113-116, 2005.

[3] M. Radulovacki, "Role of adenosine in sleep in rats," Reviews in Clinical and Basic Pharmacology, vol. 5, no. 3-4, pp. 327-339, 1985.

[4] G. A. Marks and C. G. Birabil, "Enhancement of rapid eye movement sleep in the rat by cholinergic and adenosinergic agonists infused into the pontine reticular formation," Neuroscience, vol. 86, no. 1, pp. 29-37, 1998.

[5] C. M. Portas, M. Thakkar, D. G. Rainnie, R. W. Greene, and R. W. McCarley, "Role of adenosine in behavioral state modulation: a microdialysis study in the freely moving cat," Neuroscience, vol. 79, no. 1, pp. 225-235, 1997.

[6] S. R. Ticho and M. Radulovacki, "Role of adenosine in sleep and temperature regulation in the preoptic area of rats," Pharmacology Biochemistry and Behavior, vol. 40, no. 1, pp. 33-40, 1991.

[7] R. E. Strecker, S. Morairty, M. M. Thakkar et al., "Adenosinergic modulation of basal forebrain and preoptic/anterior hypothalamic neuronal activity in the control of behavioral state," Behavioural Brain Research, vol. 115, no. 2, pp. 183-204, 2000.

[8] D. Stenberg, "Neuroanatomy and neurochemistry of sleep," Cellular and Molecular Life Sciences, vol. 64, no. 10, pp. 11871204, 2007. 
[9] M. Steriade, S. Datta, D. Paré, G. Oakson, and R. Curró Dossi, "Neuronal activities in brain-stem cholinergic nuclei related to tonic activation processes in thalamocortical systems," Journal of Neuroscience, vol. 10, no. 8, pp. 2541-2559, 1990.

[10] S. Morairty, D. Rainnie, R. McCarley, and R. Greene, "Disinhibition of ventrolateral preoptic area sleep-active neurons by adenosine: a new mechanism for sleep promotion," Neuroscience, vol. 123, no. 2, pp. 451-457, 2004.

[11] M. G. Collis and S. M. O. Hourani, "Adenosine receptor subtypes," Trends in Pharmacological Sciences, vol. 14, no. 10, pp. 360-366, 1993.

[12] P. G. Baraldi, M. A. Tabrizi, F. Fruttarolo, R. Romagnoli, and D. Preti, "Recent improvements in the development of $A_{2 B}$ adenosine receptor agonists," Purinergic Signalling, vol. 5, no. 1, pp. 3-19, 2009.

[13] S. H. Synder and P. Sklar, "Behavioral and molecular actions of caffeine: focus and adenosine," Journal of Psychiatric Research, vol. 18, no. 2, pp. 91-106, 1984.

[14] R. Sharma, S. Engemann, P. Sahota, and M. M. Thakkar, "Role of adenosine and wake-promoting basal forebrain in insomnia and associated sleep disruptions caused by ethanol dependence," Journal of Neurochemistry, vol. 115, no. 3, pp. 782794, 2010

[15] J. Melling, F. C. Belton, D. Kitching, and W. R. Stones, "Production of pure cordycepin ( 3 -deoxyadenosine) from Cordyceps militaris," Journal of Pharmacy and Pharmacology, vol. 24, p. 125, 1972.

[16] B. S. Pan, C. Y. Lin, and B. M. Huang, "The effect of cordycepin on steroidogenesis and apoptosis in MA-10 mouse leydig tumor cells," Evidence-based Complementary and Alternative Medicine, vol. 2011, Article ID 750468, 2011.

[17] N. Yoshikawa, M. Kunitomo, S. Kagota, K. Shinozuka, and K. Nakamura, "Inhibitory effect of cordycepin on hematogenic metastasis of B16-F1 mouse melanoma cells accelerated by adenosine-5' -diphosphate," Anticancer Research, vol. 29, no. 10, pp. 3857-3860, 2009.

[18] Y. J. Tsai, L. C. Lin, and T. H. Tsai, "Pharmacokinetics of adenosine and cordycepin, a bioactive constituent of cordyceps sinensis in rat," Journal of Agricultural and Food Chemistry, vol. 58, no. 8, pp. 4638-4643, 2010.

[19] Y. K. Wing, "Herbal treatment of insomnia," Hong Kong Medical Journal, vol. 7, no. 4, pp. 392-402, 2001.

[20] S. Ferré, I. Diamond, S. R. Goldberg et al., "Adenosine $A_{2 \mathrm{~A}}$ receptors in ventral striatum, hypothalamus and nociceptive circuitry: implications for drug addiction, sleep and pain," Progress in Neurobiology, vol. 83, no. 5, pp. 332-347, 2007.

[21] G. Paxinos, C. Watson, M. Pennisi, and A. Topple, "Bregma, lambda and the interaural midpoint in stereotaxic surgery with rats of different sex, strain and weight," Journal of Neuroscience Methods, vol. 13, no. 2, pp. 139-143, 1985.

[22] A. J. Lim and W. D. Winters, "A practical method for automatic real-time EEG sleep state analysis," IEEE Transactions on Biomedical Engineering, vol. 27, no. 4, pp. 212-220, 1980.

[23] F. M. Semenenko and B. M. Lumb, "Projections of anterior hypothalamic neurons to the dorsal and ventral periaqueductal grey in the rat," Brain Research, vol. 582, no. 2, pp. 237-245, 1992.

[24] L. Giménez-Llort, S. N. Schiffmann, T. Shmidt et al., "Working memory deficits in transgenic rats overexpressing human adenosine $\mathrm{A}_{2 \mathrm{~A}}$ receptors in the brain," Neurobiology of Learning and Memory, vol. 87, no. 1, pp. 42-56, 2007.
[25] S. Uchida, T. Maloney, J. D. March, R. Azari, and I. Feinberg, "Sigma $(12-15 \mathrm{~Hz})$ and delta $(0.3-3 \mathrm{~Hz})$ EEG oscillate reciprocally within NREM sleep," Brain Research Bulletin, vol. 27, no. 1, pp. 93-96, 1991.

[26] D. Menicucci, A. Piarulli, U. Debarnot, P. d'Ascanio, A. Landi, and A. Gemignani, "Functional structure of spontaneous Sleep Slow Oscillation activity in humans," PLoS ONE, vol. 4, no. 10, article e7601, 2009.

[27] R. E. Brown, R. Basheer, J. T. McKenna, R. E. Strecker, and R. W. McCarley, "Control of sleep and wakefulness," Physiological Reviews, vol. 92, no. 3, pp. 1087-1187, 2012.

[28] T. E. Bjorness, C. L. Kelly, T. Gao, V. Poffenberger, and R. W. Greene, "Control and function of the homeostatic sleep response by adenosine $A_{1}$ receptors," Journal of Neuroscience, vol. 29, no. 5, pp. 1267-1276, 2009.

[29] T. Gallopin, P. H. Luppi, B. Cauli et al., "The endogenous somnogen adenosine excites a subset of sleep-promoting neurons via $\mathrm{A}_{2 \mathrm{~A}}$ receptors in the ventrolateral preoptic nucleus," Neuroscience, vol. 134, no. 4, pp. 1377-1390, 2005.

[30] R. Basheer, R. E. Strecker, M. M. Thakkar, and R. W. McCarley, "Adenosine and sleep-wake regulation," Progress in Neurobiology, vol. 73, no. 6, pp. 379-396, 2004.

[31] S. W. Wurts and D. M. Edgar, "Caffeine during sleep deprivation: sleep tendency and dynamics of recovery sleep in rats," Pharmacology Biochemistry and Behavior, vol. 65, no. 1, pp. 155$162,2000$.

[32] D. L. Rosin, A. Robeva, R. L. Woodard, P. G. Guyenet, and J. Linden, "Immunohistochemical localization of adenosine $\mathrm{A}_{2 \mathrm{~A}}$ receptors in the rat central nervous system," Journal of Comparative Neurology, vol. 401, no. 2, pp. 163-186.

[33] D. McGinty and R. Szymusiak, "Brain structures and mechanisms involved in the generation of NREM sleep: focus on the preoptic hypothalamus," Sleep Medicine Reviews, vol. 5, no. 4, pp. 323-342, 2001.

[34] A. J. Morin and A. Beaudet, "Origin of the neurotensinergic innervation of the rat basal forebrain studied by retrograde transport of cholera toxin," Journal of Comparative Neurology, vol. 391, no. 1, pp. 30-41, 1998.

[35] J. Mariño and J. Cudeiro, "Nitric oxide-mediated cortical activation: a diffuse wake-up system," Journal of Neuroscience, vol. 23, no. 10, pp. 4299-4307, 2003.

[36] A. Nehlig, J. L. Daval, and G. Debry, "Caffeine and the central nervous system: mechanisms of action, biochemical, metabolic and psychostimulant effects," Brain Research Reviews, vol. 17, no. 2, pp. 139-169, 1992.

[37] R. W. McCarley, "Neurobiology of REM and NREM sleep," Sleep Medicine, vol. 8, no. 4, pp. 302-330, 2007.

[38] P. J. M. Van Galen, A. H. Van Bergen, C. Gallo-Rodriguez et al., "A binding site model and structure-activity relationships for the rat $\mathrm{A}_{3}$ adenosine receptor," Molecular Pharmacology, vol. 45, no. 6, pp. 1101-1111, 1994.

[39] Y. Kim, Y. Bolortuya, L. Chen et al., "Decoupling of sleepiness from sleep time and intensity during chronic sleep restriction: evidence for a role of the adenosine system," Sleep, vol. 35, no. 6, pp. 861-869, 2012.

[40] F. Okajima, H. Tomura, K. Sho, M. Akbar, M. A. Majid, and Y. Kondo, "Intracellular cross-talk between thyrotropin receptor and $\mathrm{A}_{1}$ adenosine receptor in regulation of phospholipase $\mathrm{C}$ and adenylate cyclase in COS-7 cells transfected with their receptor genes," Biochemical Journal, vol. 306, no. 3, pp. 709-715, 1995. 
[41] K. Chung-Soo, H. Ji-Yi, and K. Seunghwan, "Herbs for the tratment of insomnia," Biomoecules \& Therapeutics, vol. 19, no. 3, pp. 274-281, 2011.

[42] M. Cott, "In vitro receptor binding and enzyme inhibition by hypericum perforation extract," Pharmacopsychiatry, vol. 30, supplement 2, pp. 108-112, 1997.

[43] S. L. Yang, S. Y. Nam, J. Y. Han et al., "Alterations of spontaneous sleep architecture and cortical electroencephalogram power spectra by red ginseng extract via GABAA ergic systems," Journal of Ginseng Research, vol. 34, no. 4, pp. 304-313, 2010.

[44] S. M. Cho, M. Shimizu, C. J. Lee et al., "Hypnotic effects and binding studies for GABAA and 5-HT2C receptors of traditional medicinal plants used in Asia for insomnia," Journal of Ethnopharmacology, vol. 132, no. 1, pp. 225-232, 2010.

[45] L. Chung-Il, C. S. Kim, H. Jin-Yi et al., "Repeated administration of Korea red ginseng extract increases non-rapid-eye movement sleep via GABAAergic systems," Journal Ginseng Research, vol. 36, no. 4, pp. 403-410, 2012.

[46] E. A. Kaczka, N. R. Trenner, B. Arison, R. W. Walker, and K. Folkers, "Identification of cordycepin, a metabolite of Cordyceps militaris, as $3^{\prime}$-deoxyadenosine," Biochemical and Biophysical Research Communications, vol. 14, no. 5, pp. 456457, 1964.

[47] G. W. Zieve and E. J. Roemer, "Cordycepin rapidly collapses the intermediate filament networks into juxtanuclear caps in fibroblasts and epidermal cells," Experimental Cell Research, vol. 177, no. 1, pp. 19-26, 1988. 




The Scientific World Journal
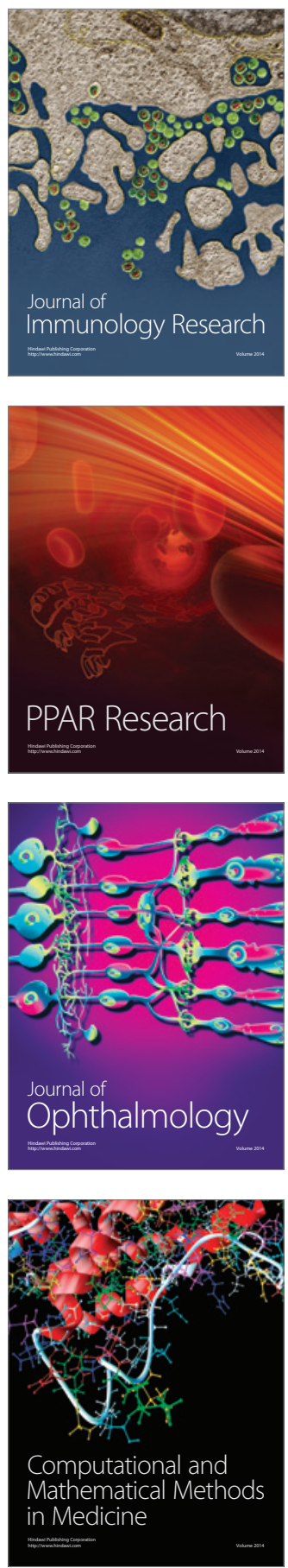

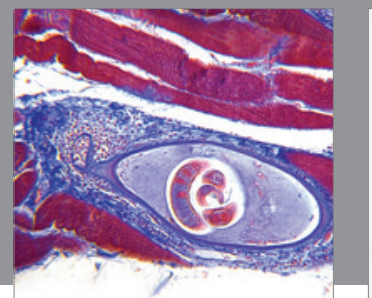

Gastroenterology

Research and Practice
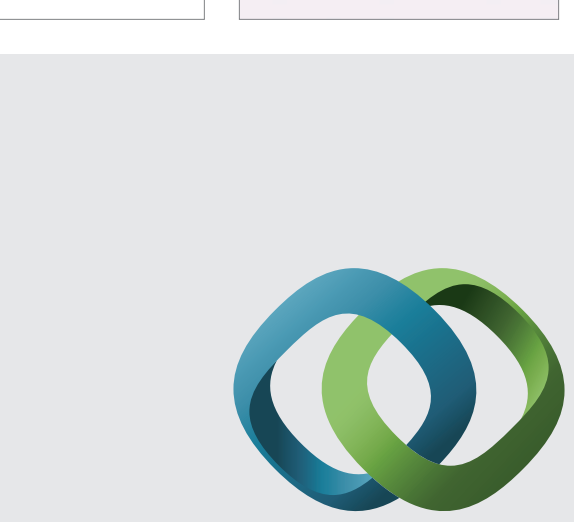

\section{Hindawi}

Submit your manuscripts at

http://www.hindawi.com
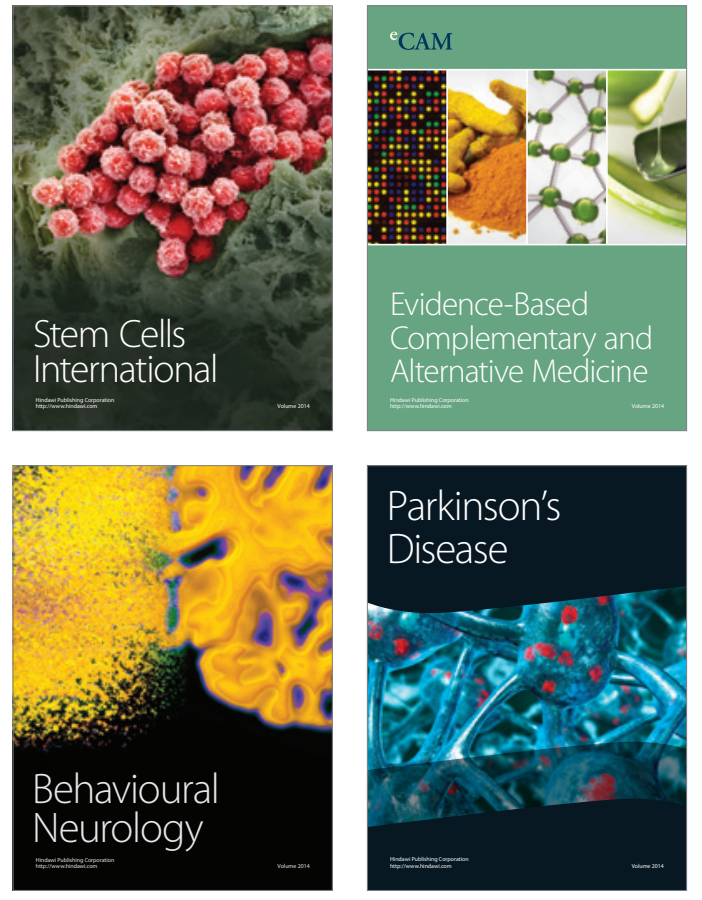
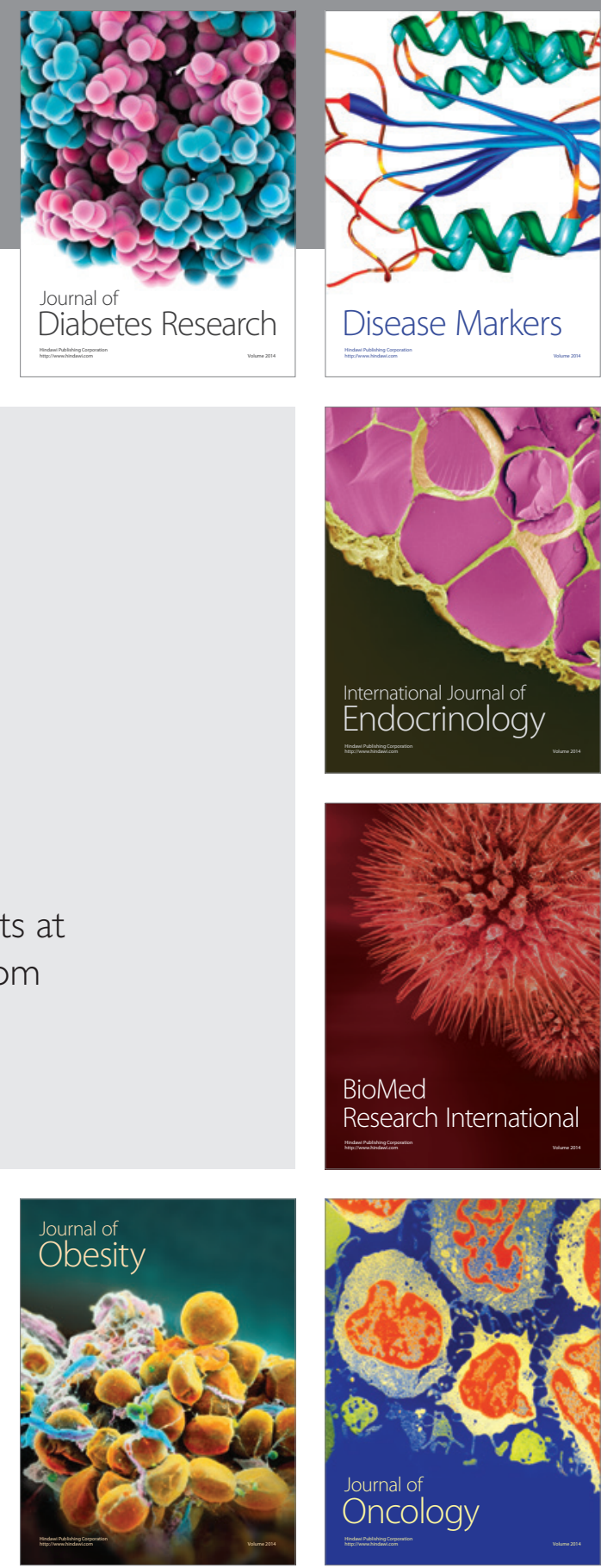

Disease Markers
